\title{
Phase center variations problem in GPS/GLONASS observations processing
}

\author{
Karol Dawidowicz \\ University of Warmia and Mazury, Institute of Geodesy, ul. Oczpowskiego 1, 10-719 Olsztyn, Poland
}

\begin{abstract}
It is generally known that the phase center of a GNSS antenna is not a stable point. For any given GNSS antenna, the phase centers will change with the changing direction of the signal from a satellite. The problem is particularly important when different antennae are used in measurements. Ignoring these phase center variations in measurements can lead to serious vertical errors. The way to avoid these errors is by applying antenna phase center variation models in processing. Three major methods are presently available to determine mean antenna phase center offsets as well as variations of the phase center with elevation and azimuth for GNSS receiver antennas. Because of this and because of some problems in introducing of absolute models at present we can speak of three models of receiver antennas PCV (relative, absolute converted and absolute) and two of satellites antennas (standard and absolute). Additionally, processing simultaneously observations from different positioning systems e.g. GPS and GLONASS, we can expect a further complication resulting from different structure of signals and differences in satellites constellations.

The aim of this paper is to study the height differences in short static GPS/GLONASS observations processing when different calibration models are used. The analysis was done using 24 hour GNSS data divided by one hour observation sessions. The results show that switching between different PCV models may have a visible effect on height determination, particularly in high accuracy applications.
\end{abstract}

Keywords: PCV, Global Positioning System (GPS), GLONASS, Global Navigation Satellite System (GNSS) antennas.

\begin{tabular}{|ll|}
\hline Nomenclature \\
$\mathrm{R}$ & relative antenna phase center variations model \\
$\mathrm{A}$ & absolute GPS-only antenna phase center variations model \\
$\mathrm{A}+$ & absolute GPS+GLONASS antenna phase center variations model \\
\hline
\end{tabular}

\section{Introduction}

The position of electrical antenna phase center of an individual measurement and the position of the mean electrical antenna phase center are not identical. The deviations, as a result of this comparison, are defined as antenna phase center variations (PCV).

GNSS antenna phase center can exhibit shifts in position with varying observed elevation angle and azimuth to the satellite e.g. [16], [22]. The phase centre also varies based on the frequency of the received signals. Because the computed baseline vector is a relative measurement between two antennae, over short baselines phase centre variations will cancel if identical antennae are used and the antennae are orientated to the same direction. The effect will not cancel in situations where different antennae are used at either end of a baseline. Neither will antenna phase centre motion cancel over very long baselines. As the magnitude of phase centre variations can be in the order of several centimeters [4], [16], this problem can become significant for applications requiring the highest attainable precision from GNSS [3]. A review of the antenna phase center variations problem can be found e.g. in [1], [5], [8], [10], [14], [17], [18], [19].

PCV are determined by the calibration process and then antenna phase center corrections models can be created. Actually three main methods have been distinguished to determine GNSS antenna phase center variations:

- relative field calibrations,

- anechoic chamber measurements,

- absolute field calibrations. 
A review of the antenna phase center variations modeling methods can be found e.g. in [7], [9], [11-13], [15], [17], [23].

Because still there are no results of absolute calibrations for all antenna types, for these antennas, absolute models are created through the conversion from relative. Thus, at present there are available three models of receiver antennas and two GNSS satellites. For receiver antennas, there is:

- the relative IGS antenna phase center correction model from relative field calibration,

- the absolute IGS antenna phase center correction model (converted from relative) - all the relative antenna offsets and phase center variations that the National Geodetic Survey has computed have been added to the absolute values for AOAD/M T antenna,

- the absolute IGS antenna phase center correction model from absolute field calibration or from anechoic chamber measurements.

For GNSS satellites, there may be two solutions:

- the standard IGS antenna offset model,

- the absolute antenna phase center correction model.

So many potential models may introduce some confusion. Additionally, processing simultaneously observations from different positioning systems e.g. GPS and GLONASS, we can expect a further complication resulting from different structure of signals and differences in satellites constellations (GLONASS satellites complete an orbit in approximately 11 hours 15 minutes while GPS satellites in approximately 11 hours 58 minutes). Another difficulty is the fact that different GLONASS satellites transmit signals on different frequencies. A review of the GPS/GLONASS observations processing problem can be found e.g. in [2], [6], [20]. In author opinion however it is lack of similar studies on the PCV problem.

GLONASS satellites transmit signals using Frequency Division Multiple Access (FDMA) and have available 12 allocated radio frequency carriers in L1 frequency band and 12 carriers in L2 band. Each satellite transmits signals at two frequencies, using one assigned radio frequency carrier in L1 frequency band and one assigned carrier in L2 frequency band [10].

The GLONASS PCV calibration differs compared to GPS. The reason is the different frequencies of individual GLONASSS satellites. The GLONASS constellation was for a long time not sufficient to perform a PCV calibration during the first absolute field calibration, the robot was stopped after three complete days without sufficient coverage of the antenna hemisphere. However with the current constellation several calibrations for different GNSS antenna types have been executed. Because the absolute robot calibration estimated PCV from the mixture of observed GLONASS frequencies the calibration are satellite constellation dependent and are expected to be not as accurate as for GPS [21].

An alternative PCV modeling has been developed, which allows frequency dependent GLONASS PCV determined. Fundamental assumption of the model is linearity of PCV changes for GPS/GLONASS and GLONASS/GLONASS frequencies [16]. In the last resort it is common to use GPS PCV for the correction of GLONASS PCV in lack of better information. Still several issues related to GLONASS PCV are pressing and important to investigate.

The aim of this paper is to study the height differences in short static GPS/GLONASS observations processing when different calibration models are used. The analysis was done using 24 hour GNSS data divided by one hour observation sessions. The results show that switching between different PCV models may have a visible effect on height determination, particularly in high accuracy applications.

\section{Research area}

Analysis were done based on the observations conducted on three points. Antenna and receiver types on selected points are presented in Table 1. As fixed the point with antennae JAV_GRANT-G3T was selected.

Table 1. Hardware on selected points

\begin{tabular}{llll}
\hline Point name & KROL & 0001 & 0002 \\
\hline Antenna type & JAVAD RINGANT G3T & TPSHIPER_PLUS & TPSHIPER_PLUS \\
Localization & & & \\
Receiver type & JAVAD TR_G3TH SIGMA & Hiper Pro & Hiper Pro \\
\hline
\end{tabular}


Point KROL is the ASG-EUPOS reference station located in Olsztyn. On that point there were optimal observing conditions without any obstructions. Points 0001 and 0002 are marked in an urban area, where trees or buildings limit the number of observed. Satellites visibility during the observations is presented on Figure 1.

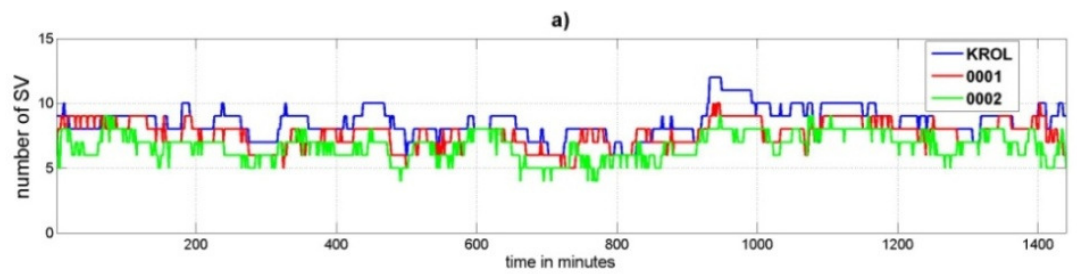

b)

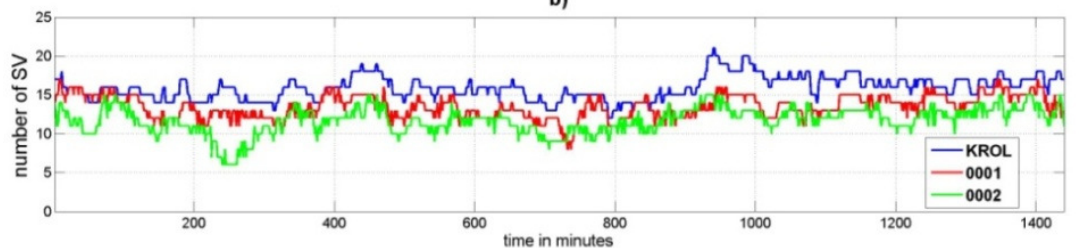

Fig. 1. Satellites visibility on measured points: a) GPS only, b) GPS+GLONASS

Such points location gave additional possibility to analyze the height differences when different calibration models are used depending on satellites visibility on points

The selected antennas are characterized by relatively small differences between their phase characteristics and the changes in these characteristics for subsequent types of calibration. A comparison of the antenna phase characteristics on the measured points is shown in Figs. 2-3.

a)

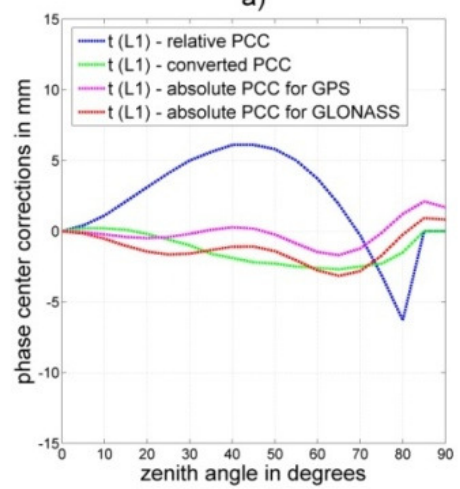

b)

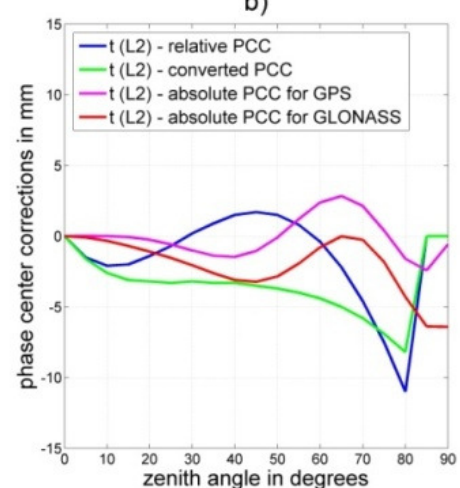

Fig. 2. IGS zenith angle dependent phase center variations for antenna JAVAD RINGANT G3T: a) for L1 frequency, b) for L2 frequency

a)

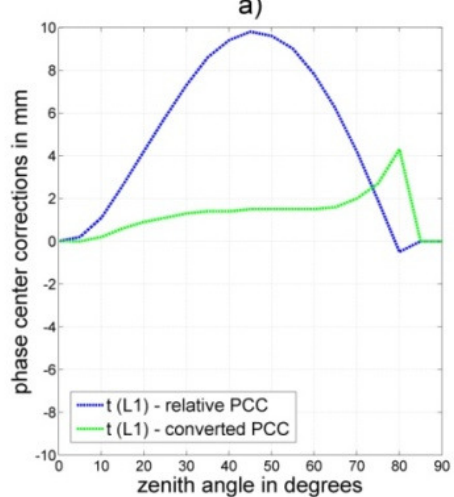

b)

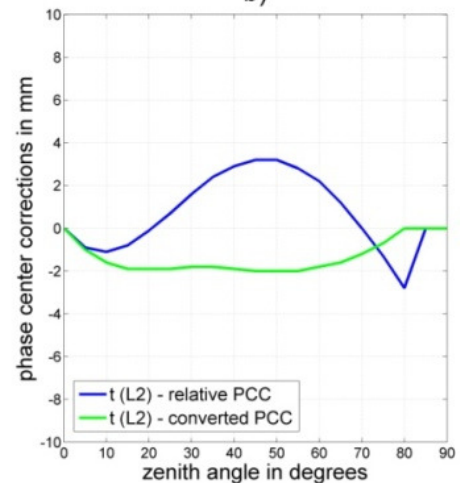

Fig. 3. IGS zenith angle dependent phase center variations for antenna TPSHIPER_PLUS: a) for L1 frequency, b) for L2 frequency 
It is visible that two selected antennas have small differences in their PCV profiles. Generally, the greatest difference was observed for medium zenith angles (from $30^{\circ}$ to $60^{\circ}$ ). These differences are also visible in comparing PCV characteristics for the same antenna, obtained from different calibration procedures (especially between relative and absolute calibration results) - the maximum differences for the same frequency reach $10 \mathrm{~mm}$. Comparing absolute elevation dependent PCV for GPS and GLONASS signals (Figs. 1) there are only small 2-3 mm differences visible.

In addition to the above figures, the L1 and L2 offsets (PCO) for the used antennas are presented (Table 2). Clear differences were also found, especially between the results of the relative and the other two calibration methods.

Table 2. Offsets for selected antennas

\begin{tabular}{|c|c|c|c|c|c|c|}
\hline \multirow[t]{3}{*}{ Antenna type } & \multicolumn{6}{|c|}{ Offsets (PCO) [mm] } \\
\hline & \multicolumn{2}{|c|}{ relative field calibration } & \multicolumn{2}{|c|}{ converted from relative } & \multicolumn{2}{|c|}{ absolute field calibration } \\
\hline & LI & $L 2$ & $L 1$ & $L 2$ & Ll & $L 2$ \\
\hline JAV_GRANT-G3T & 69.4 & 60.6 & 50.6 & 52.7 & 50.3 & 46.8 \\
\hline TPSHIPER PLUS & 105.9 & 97.1 & 87.1 & 89.2 & - & - \\
\hline
\end{tabular}

Analyses were based on one-day 24-hour observation sessions (20.11.2012). The following GNSS parameters were assumed for observations: sampling interval $1 \mathrm{~s}$, minimum satellite elevation $10^{\circ}$. All 24-hour observations were divided into 1 hour sessions and processed in a single-baseline mode. To post-processing a commercial software enabling the introduction (definition) of PCC models themselves was used. It should be noted, that when we used relative calibration model GPS PCV are adopted for the correction of GLONASS PCV because of lack of better information. Additionally for TPSHIPER_PLUS antenna there are only absolute-converted PCV model available (igs08.atx) without GLONASS PCV corrections. This cause also adopting GPS PCV corrections for GLONASS signals in post-processing.

Processing was done in three main variants (STRATEGIES):

- using the relative IGS models (relative field calibration - R);

- using absolute GPS-only IGS models (absolute field calibration or converted for TPSHIPER PLUS antenna - A).

- using absolute GPS+GLONASS IGS models (A+).

Each "strategy' was processed using L1 and L2 phase observations and the so-called "ionosphere-free linear combination" - L3. When we form L3 combination, the ionospheric path delay is virtually eliminated. Using the L3 combination in our study causes the differences in antenna PCV of both frequencies to appear in the final results. All other processing options (tropospheric model, orbits, satellite antenna calibrations, etc.) were identical in all runs. The results of calculation and their discussion are provided below.

\section{Results}

The paper presents the height differences obtained in short GPS/GLONASS observations processing when different calibration models are used and different observing conditions were on measured points. The baseline results obtained with the JAV_GRANT G3T and TPSHIPER_PLUS antennas (height differences for previously mentioned processing strategies, on the 0001 and 0002 points) are presented on Figs 4 and 5. Additionally in table 3 there are presented a summary of the height differences obtained for two baselines.

In analyzing the results obtained for the GPS-only solutions (Fig. 4) it can be seen that the height differences for the GPS-only solutions are within $1.0 \mathrm{~cm}$ (L1 and L2 processing variants) and $1.5 \mathrm{~cm}$ (L3 processing variant). As expected, using absolute GPS/GLONASS IGS models did not affect the results: height obtained using $\mathbf{A}$ and $\mathbf{A}+$ mode were the same. Additionally it is evident that visibility of satellites affect the final results - the heights differences on the two test points visible differ (especially for L3 processing variant).

Significantly larger differences were obtained for processing done using GPS/GLONASS observations (Fig. 5). Height differences are generally within $2.0 \mathrm{~cm}$ for L1 and L2 processing variants and $3.0 \mathrm{~cm}$ for L3 processing variant. In that variant using absolute GPS/GLONASS IGS models visible affect the results: height obtained using $\mathbf{A}$ and $\mathbf{A}+$ mode differ. As previously the visibility of satellites affect the final results - the heights differences on the two test points visible differ. 

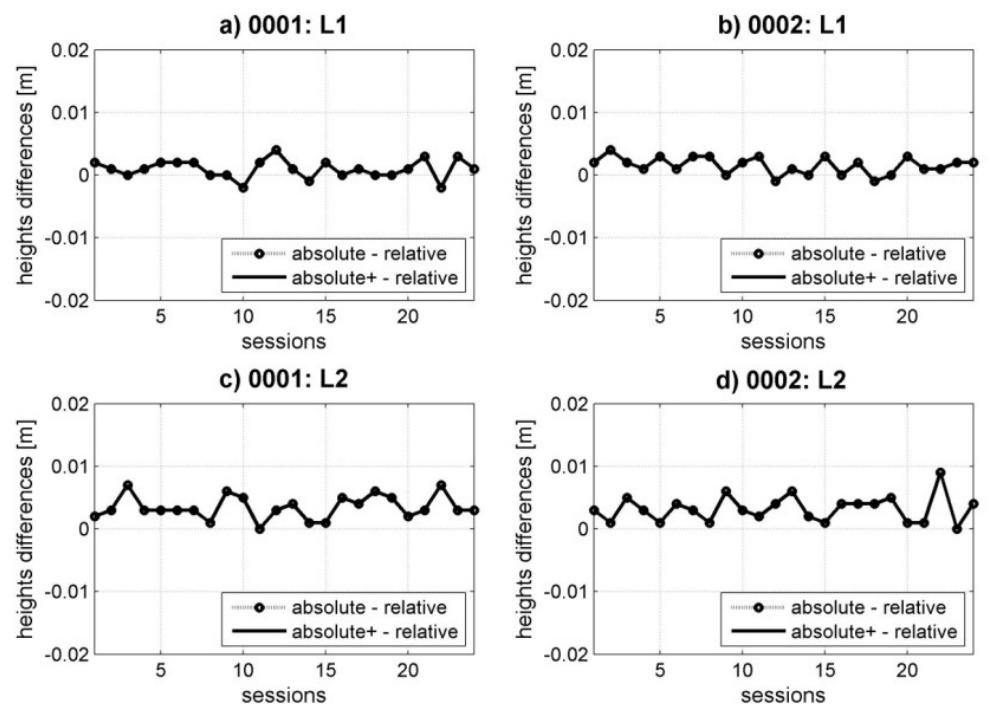

e) 0001: L3
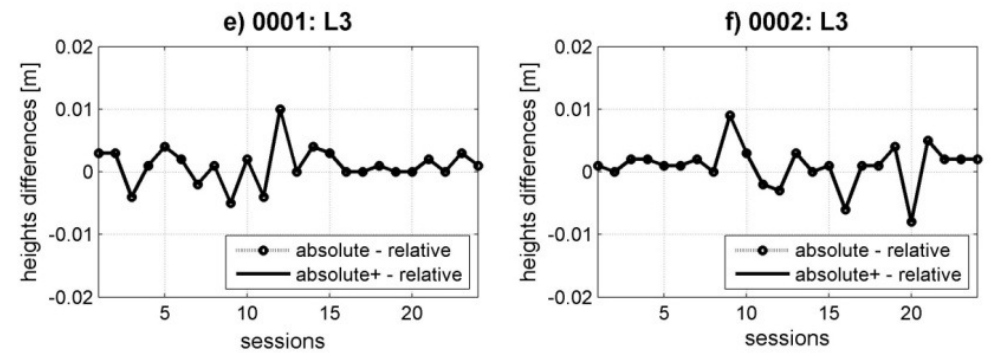

Fig. 4. Height differences obtained from the processing of GPS-only observations using absolute or absolute+ and relative PCC models (absolute - relative)
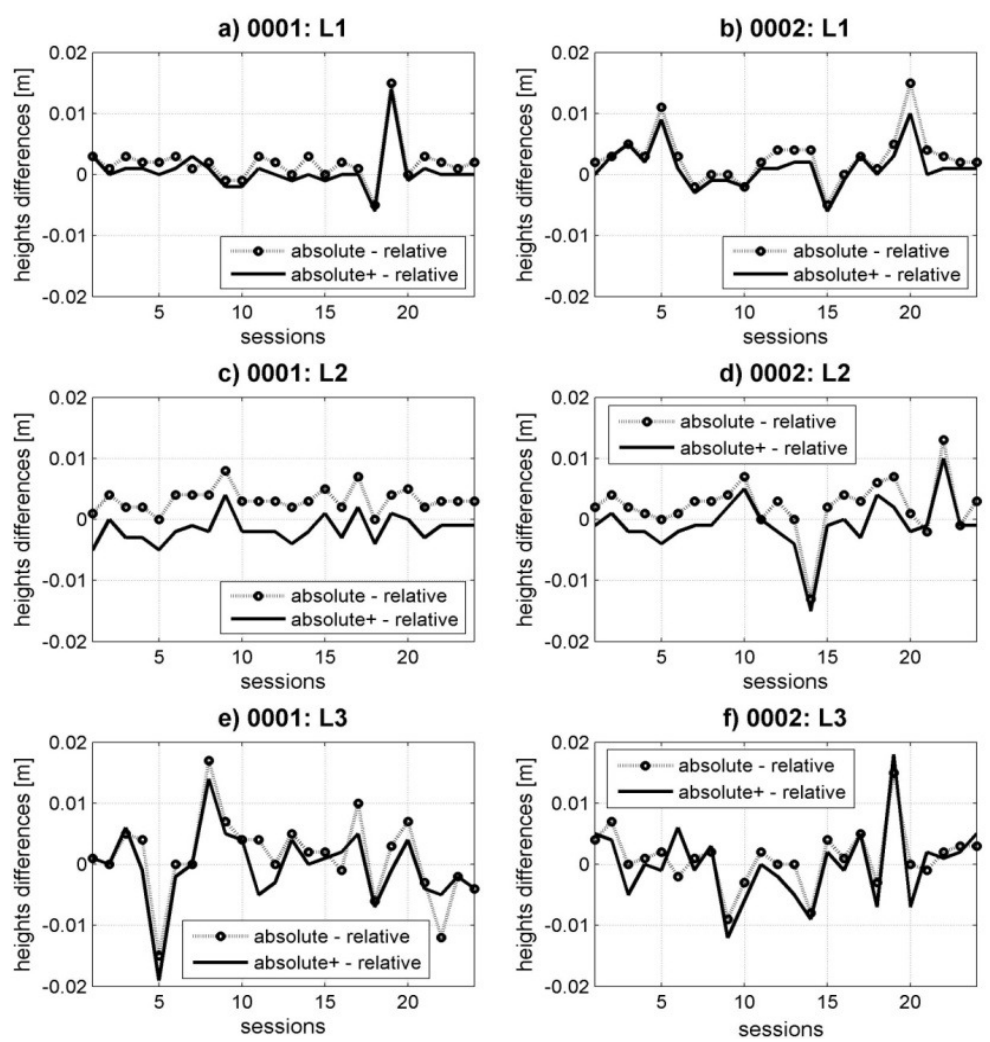

Fig. 5. Height differences obtained from the processing of GPS/GLONASS observations using absolute or absolute + and relative PCC models (absolute - relative) 
Table 3. Summary of height differences obtained for 0001 and 0002 [m]

\begin{tabular}{|c|c|c|c|c|c|c|}
\hline \multirow{2}{*}{$\begin{array}{l}\text { Type of } \\
\text { observations }\end{array}$} & \multicolumn{3}{|c|}{ Height differences for $\mathbf{0 0 0 1}$ point } & \multicolumn{3}{|c|}{ Height differences for $\mathbf{0 0 0 2}$ point } \\
\hline & Max. & Min. & Average & Max. & Min. & Average \\
\hline & \multicolumn{6}{|c|}{ GPS-only solutions $(\mathbf{A}-\mathbf{R}$ and $\mathbf{A}+-\mathbf{R})$} \\
\hline L1 & 0.004 & -0.002 & 0.001 & 0.004 & -0.001 & 0.002 \\
\hline $\mathrm{L} 2$ & 0.007 & 0 & 0.003 & 0.009 & 0 & 0.003 \\
\hline \multirow[t]{2}{*}{ L3 } & 0.010 & -0.005 & 0.001 & 0.009 & -0.008 & 0.001 \\
\hline & \multicolumn{6}{|c|}{ GPS/GLONASS solutions $(\mathbf{A}-\mathbf{R})$} \\
\hline L1 & 0.003 & -0.005 & 0.002 & 0.015 & -0.005 & 0.003 \\
\hline $\mathrm{L} 2$ & 0.008 & 0 & 0.003 & 0.007 & -0.013 & 0.002 \\
\hline \multirow[t]{2}{*}{ L3 } & 0.017 & -0.015 & 0.001 & 0.015 & -0.009 & 0.001 \\
\hline & \multicolumn{6}{|c|}{ GPS/GLONASS solutions $(\mathbf{A}+-\mathbf{R})$} \\
\hline L1 & 0.014 & -0.006 & 0.001 & 0.010 & -0.006 & 0.001 \\
\hline L2 & 0.004 & -0.005 & -0.002 & 0.010 & -0.015 & -0.001 \\
\hline L3 & 0.014 & -0.019 & 0 & 0.018 & -0.012 & 0 \\
\hline
\end{tabular}

Comparing the minimum and maximum height differences for GPS-only and GPS/GLONASS solutions (Table 3), it is clear that the effect of switching between calibration models for GPS/GLONASS results, in the presented case, is stronger. Additionally, as expected, differences in visibility of satellites clearly affect the final results - there are visible evident differences in Max. and Min. values on the two test points. Average height differences, on the other hand, are small and do not exceed in any case $3 \mathrm{~mm}$. As expected, height changes being a result of changes in the satellites' constellation above the point of measurement for longer periods of time averaged.

Generally it can be concluded that GPS-only results are comparable to results obtained in other studies e.g. [5], [19]. There are lack of similar studies about GPS/GLONASS observations processing. The large jumps in the vertical component in GPS/GLONASS results, in author opinion, are worth of further study.

Finally, it should be noted that the obtained height differences are the result of switching from the relative to the absolute PCV model for two selected types of antennas - for other antennas results can differ.

\section{Conclusions}

In this study, the height differences caused by using different calibration models in GPS-only and GPS/GLONASS observations processing in dependence of satellite visibility on points were compared. The analysis was conducted using 24 hour GNSS data, collected with two different types of receivers and antennas, divided into one hour observation sessions.

The update of receiver antenna calibrations from relative to absolute in our study (using GPS-only observations in one hour sessions) induces a jump (depending on the types of observations and measurements conditions on point) in the vertical component from $0.5 \mathrm{~cm}$ to $1.7 \mathrm{~cm}$. These jumps are relevant for many high accuracy applications.

For GPS/GLONASS observations differences are significantly larger $(0.8 \mathrm{~cm}$ to $3.3 \mathrm{~cm}$. In author opinion this problems need further study. Next analysis are planed using more antenna types and different software.

\section{References}

[1] Braun, J.; Rocken, C.; Meertens, C. M.; Johanson, J. 1993. GPS antenna mixing and phase center corrections, Eos Trans. AGU, Fall Meeting Supplement: 197

[2] Bruninx, C. 2007. Comparing GPS-only with GPS+GLONASS positioning in a regional permanent GNSS network, GPS Solutions 11(2): 97-106 http://dx.doi.org/10.1007/s10291-006-0041-9

[3] Cellmer S. 2012. A Graphic Representation of the Necessary Condition for the MAFA Method, Transactions on Geoscience and Remote Sensing 50(2): 482-488. http://dx.doi.org/10.1109/TGRS.2011.2161321

[4] Dawidowicz, K. 2010. Antenna phase center variations corrections in processing of GPS observations with use of commercial software, Technical Sciences 13: 120-132. http://dx.doi.org/10.2478/v10022-010-0012-9

[5] Dawidowicz, K. 2013. Impact of different GNSS antenna calibration models on height determination in the ASG-EUPOS network - a case study, Survey Review 45(332): 386-394. http://dx.doi.org/10.1179/1752270613Y.0000000043 
[6] Dodson, A. H.; Moore, T.; Baker, F. D.; Swann, J. W. 1999. Hybrid GPS+GLONASS, GPS Solutions 3(1): 32-41. http://dx.doi.org/10.1007/PL00012777

[7] Falko, M.; Seeber, G.; Völksen, Ch.; Wübbena, G.; Schmitz, M. 1998. Results of Absolute Field Calibration of GPS Antenna PCV, ION GPS-98 Proceedings of the 11th International Technical Meeting of the Satellite Division of the Institute of Navigation, Nashville, TN; UNITED STATES; 1518 Sept., 31-38.

[8] Geiger, A. 1998. Modeling of Phase Center Variation and its Influence on GPS Positioning, Proceedings of the International GPS-Workshop Darmstadt, April 10 to 13, Editor: Erwin Groten, Robert Strauß, Lecture Notes in Earth Sciences 19: 210-222.

[9] G $\square$ rres. B.; Campbell. M.; Becker, M.; Siemes, M. 2006. Absolute calibration of GPS antennas: Laboratory results and comparison with field and robot techniques, GPS solutions 10: 136-145.

[10] Hofmann-Wellenhof, B.; Lichtenegger, H.; Wasle, E. 2008. GNSS - Global Navigation Satellite Systems, Springer-Verlag Wien, Austria, 516 p.

[11] Mader, G. L. 1999. GPS Antenna Calibration at the National Geodetic Survey, GPS Solutions 3(1): 50-58. http://dx.doi.org/10.1007/PL00012780

[12] Rothacher, M. 2001. Comparison of Absolute and Relative Antenna Phase Center Variations, GPS Solutions 4: 55-60. http://dx.doi.org/10.1007/PL00012867

[13] Rothacher, M.; Mader, G. 1996. Combination of antenna phase center offsets and variation: antenna calibration set IGS_01, anonymous ftp ubeclu.unibe.ch.

[14] Rocken, C. 1992. GPS antenna mixing problems, UNAVACO Memo, November 12.

[15] Schupler, B. R.; Clark, T. A. 1994. Signal Characteristics of GPS User Antennas, Navigation, Journal of the Institute of Navigation 41(3): $277-295$.

[16] Schupler, B. R.; Clark, T. A. 2001. Characterizing the Behavior of Geodetic GPS Antennas, GPSWorld February, 48-55.

[17] Schmid, R.; Rothacher, M.; Thaller, D.; Steigenberger, P. 2005. Absolute phase center corrections of satellite and receiver antennas, GPS Solutions 9(4): 283-293. http://dx.doi.org/10.1007/s10291-005-0134-x

[18] Schmitz, M.; Wübbena, G.; Boettcher, G. 2002. Tests of phase center variations of various GPS antennas, and some results. GPS Solutions 6: 18-27. http://dx.doi.org/10.1007/s10291-002-0008-4

[19] V $\square$ lksen, Ch. 2006. The Impact of different GPS Antenna Calibration Models on the EUREF Permanent Network, Report on the Symposium of the IAG Sub-commission for Europe (EUREF), mitteilungen des BKG, Band 38, Verlag des BKG, Frankfurt/Main: 73-78.

[20] Weber, R.; Slater, J. A.; Fragner, E.; Glotov, V.; Habrich, H.; Romero, I.; Schaer, S. 2005. Precise GLONASS Orbit Determination within the IGS/IGLOS Pilot Project, Advances in Space Research 36: 369-375. http://dx.doi.org/10.1016/j.asr.2005.08.051

[21] Wübbena, G.; Schmitz, M.; Boettcher, G.; Schumann, Ch. 2006. Absolute GNSS Antenna Calibration with a Robot: Repeatability of Phase Variations, Calibration of GLONASS and Determination of Carrier-to-Noise Pattern, Proceedings of the IGS Workshop: Perspectives and Visions for 2010 and beyond, 8-12 May 2006, Darmstadt, Germany.

[22] Wu, J. T.; Wu, S. C.; Hajj, G. A.; Bertiger, W. I.; Lichten, S. M. 1993. Effects of antenna orientation on GPS carrier phase, Manuscripta Geodetica 18: 91-98.

[23] Zeimetz, P.; Kuhlman, H. 2008. On the Accuracy of Absolute GNSS Antenna Calibration and the Conception of a New Anechoic Chamber, in FIG Working Week 2008, Stockholm, Sweden 14-19 June. 